\title{
Rapid and Sensitive Detection of Troponin I-T-C Complex from Human Serum using Microwave-Accelerated Metal-Enhanced Fluorescence
}

\author{
Kadir Aslan* \\ Morgan State University, Department of Chemistry, 1700 East Cold Spring Lane, Baltimore, MD 21251. \\ * Corresponding author: kadir.aslan@morgan.edu (Kadir Aslan)
}

\begin{abstract}
We present the rapid and sensitive detection of Troponin I-T-C (Tn I-T-C) complex from buffer and human serum samples using MicrowaveAccelerated and Metal-Enhanced Fluorescence (MA-MEF) technique, which is based on the combined use of low power microwave heating, silver nanoparticle films (SNFs) and fluorescence spectroscopy. The detection of Tn I-T-C complex from buffer solutions and human serum samples on SNFs was carried out using fluorescence-based immunoassays at room temperature (control immunoassay, 2 hour total assay time) and using lowpower microwave heating (MA-MEF-based immunoassay, 1 minute total assay time). A lower detection limit for Tn I-T-C complex from buffer solutions in the control immunoassay and MA-MEF-based immunoassay was $0.01 \mathrm{ng} \mathrm{ml}^{-1}$ and $0.005 \mathrm{ng} \mathrm{ml}^{-1}$, respectively. However, the lower detection limit for Tn I-T-C complex from human serum in the control immunoassay was increased to $10 \mathrm{ng} \mathrm{ml}^{-1}$. The use of MA-MEF technique afforded for the detection of Tn I-T-C complex from human serum samples in 1 min with a lower detection limit of $0.05 \mathrm{ng} \mathrm{ml}^{-1}$.
\end{abstract}

Keywords: Troponin I-T-C, Immunoassays, Cardiac Markers, Silver Nanoparticles, Surface Plasmon Resonance, Plasmon Controlled Fluorescence, Metal-Enhanced Fluorescence, Microwave Heating

Citation: K. Aslan, et al. Rapid and Sensitive Detection of Troponin I-T-C Complex from Human Serum using Microwave-Accelerated MetalEnhanced Fluorescence. Nano Biomed. Eng. 2011, 3(3), 179-183. DOI: 10.5101/nbe.v3i3.p179-183.

\section{Introduction}

Cardiovascular diseases, such as myocardial infarction (AMI), are among the leading causes of mortality in developed countries. Several conventional cardiac markers are used for the assessment of an AMI [1], such as myoglobin, troponin I (cTnI), troponin $\mathrm{T}$ (cTnT) and CK-MB [2]. Previous studies have proven that troponin is more sensitive and significantly more specific in diagnosing AMI than myglobin or CK-MB and cTnI and TnT can be used as reliable markers for cardiac risk assessment [3].

It is also known that troponin exists as a complex between the three single chain polypeptides [4]: 1) cTnT (binds the other troponin components to tropomyosin), 2) cTnI (inhibits ATP activity when bound to actin), 3) troponin $\mathrm{C}$ (cTnc, contains binding sites for calcium). Most of the troponin is found as this three unit complex but there is also a small percentage $(<2-8 \%)$ of unbound troponin in the cytoplasm of the cardiac muscle cell. When myocyte damage occurs, troponin is released into the serum both as the individual troponin proteins I, T, and $\mathrm{C}$ and as binary and tertiary complexes such as TC and ITC [5]. cTnI-TnC complex formation is considered to improve the stability of cTnI. cTnI, which is extremely unstable in its free form. The stability of cTnI is significantly improved in binary complex formed with TnC or in the ternary complex cTnI-cTnT-TnC [6]. In this regard, one can potentially better assess AMI by testing the human serum samples for these troponin complexes.

Our research group has previously reported the rapid detection of TnI from human whole blood samples using MA-MEF technique [7]. In this previous paper [7], the detection of TnI from buffer samples was first carried out using a sandwich-type immunoassay on SNFs at room temperature (control immunoassay) and using microwave heating (MA-MEF-based immunoassay), where the lower detection limit for TnI was $0.1 \mathrm{ng} \mathrm{ml}^{-1}$ and $0.005 \mathrm{ng} \mathrm{ml}^{-1}$, respectively. The total assay time was 2 hours and 1 minute for immunoassays run at room temperature and using microwave heating, respectively. Whole blood immunoassay run on SNFs using microwave heating 
yielded Tn I-concentration dependent fluorescence emission, where as low as $0.05 \mathrm{ng} \mathrm{ml}^{-1}$ of TnI was detected within 1 minute (total assay time) [7].

In this paper, we report the rapid and sensitive detection of Tn I-T-C complex from buffer and human serum samples using MA-MEF technique. In this regard, SNFs were deposited onto glass slides using Tollen's reaction scheme. The detection of Tn I-T-C complex from buffer solutions and human serum samples on SNFs was carried out using fluorescence-based immunoassays at room temperature (control immunoassay and using low-power microwave heating (MA-MEF-based immunoassay). The lower detection limit for Tn I-T-C complex from buffer solutions in the control immunoassay and MA-MEF-based immunoassay was determined to be $0.01 \mathrm{ng} \mathrm{ml}^{-1}$ and $0.005 \mathrm{ng} \mathrm{ml}^{-1}$, respectively. The lower detection limit for Tn I-T-C complex from human serum in the control immunoassay was increased to $10 \mathrm{ng} \mathrm{ml}^{-1}$. The use of MA-MEF technique afforded for the detection of Tn I-T-C complex from human serum samples in $1 \mathrm{~min}$ with a lower detection limit of $0.05 \mathrm{ng} \mathrm{ml}^{-1}$.

\section{Materials and methods}

\subsection{Materials}

Protein A, human serum, bovine serum albumin (BSA), silver nitrate $(99.9 \%)$, trisodium citrate, phosphate buffered saline (PBS), press-to-seal silicone isolators ( 8 well, depth $\times$ diameter: $1.0 \mathrm{~mm} \times 9 \mathrm{~mm}$ ) and silaneprepTM glass (amine-modified) slides were purchased from Sigma-Aldrich. Relevant antibodies (capture and FITC-labeled detection antibody), standard solutions of Troponin I-T-C complex was purchased from Advanced Immunochemical, Inc. (CA, USA). All chemicals were used as received.

\subsection{Methods}

Coating of amine-modified glass slides with silver nanoparticle films (SNFs) were carried out using a 6-step Tollen's reaction scheme:

1) a solution of silver nitrate $(0.5 \mathrm{~g}$ in $60 \mathrm{ml}$ of deionized water) was placed in a clean $100-\mathrm{ml}$ glass beaker with a magnetic stirrer.

2) While stirring the silver nitrate solution at speed 10 (on a Fisher Heater/Stirrer), $200 \mu \mathrm{L}$ of freshly prepared $5 \%$ $(\mathrm{w} / \mathrm{v})$ sodium hydroxide solution is added. This results in the formation of dark brown precipitates of silver particles in solution.

3) The precipitates were immediately re-dissolved by 2 $\mathrm{ml}$ ammonium hydroxide.

4) The resultant clear solution is cooled down to $5^{\circ} \mathrm{C}$ by placing the beaker in an ice bath, followed by soaking the amine-modified glass slides in the solution.

5) After $2 \mathrm{~min}$, a fresh solution of D-glucose ( $0.72 \mathrm{~g}$ in 15 $\mathrm{ml}$ of water) is added.

6) Subsequently, the temperature of the mixture is warmed to $30^{\circ} \mathrm{C}$. As the color of the mixture turns from "yellow-green" to "yellow-brown" and the color of the amine-modified glass slides become green (in $2 \mathrm{~min}$ ),

SNFs-deposited amine-modified glass slides are removed from the mixture. SNFs were rinsed with deionized water and sonicated for 30 seconds at room temperature. SNFs were then rinsed with deionized (DI) water several times and air-dried and kept in an air-tight vessel until further use.

The construction of the MA-MEF-based immunoassay and control immunoassay for Tn I-T-C complex is depicted in Fig. 1. The immunoassay procedure was divided into two parts:

1. Pre-assay (Fig. 1A), where protein A, capture antibody and BSA (all in $\mathrm{pH}=7.4$, PBS buffer) attached to SNFs in sequence by incubation at room temperature for 30 minutes for each step,

2. MA-MEF assay (Fig. 1B), where Tn I-T-C complex (in buffer or serum) and detector antibody (in buffer) is incubated on SNFs using microwave heating for 30 seconds. As a control immunoassay, these steps were also separately carried out at room temperature (60 minutes each step).

The most relevant steps in the MA-MEF-based immunoassay for Tn I-T-C complex using microwave heating are the steps involving Troponin I-T-C complex and detector antibody incubation on SNFs. In this regard, one can prepare "pre-assay" slides at any time and store for future use in MA-MEF based immunoassay. In both immunoassays (control and MA-MEF-based), Troponin I-T-C complex was present either only in buffer in human serum. In the immunoassays run for human serum samples, a solution of Tn I-T-C complex in phosphate buffer $(\mathrm{pH}=7)$ was mixed with human serum $(50 \% \mathrm{v} /$ $\mathrm{v}$ mixture; final volume: $50 \mu \mathrm{l}$; final concentration range for TnI: $0.001-100 \mathrm{ng} \mathrm{ml}^{-1}$ ). These mixtures or a buffer solution (control sample, no Troponin I-T-C complex) was placed inside the wells of silicon isolators and the SNFs were heated for 30 seconds in a commercially available microwave oven (Emerson, maximum power $700 \mathrm{~W}$ microwave oven, Model: MW8784B, power setting 3 was used). The unbound material was removed by rinsing with phosphate buffer three times. Then, $50 \mu \mathrm{l}$ of $10 \mu \mathrm{M}$ FITClabeled detection antibody was subsequently added to the wells and heated for 30 seconds in the microwave cavity, followed by rinsing with buffer to remove the unbound material.

Fig. 2A shows the real-color image of SNFs with 8 -well silicon isolator that affords for the processing of 8 different samples at once. Fig. 2B shows a photograph of the experimental geometry used for the measurement of fluorescence emission from the immunoassays. In this regard, SNFs were placed horizontally above a laser table on an optical post. The excitation source (473 nm continuous wave laser (BW\&Tek, Inc. Delaware, USA) is reflected at a $45^{\circ}$ angle to the wells on SNFs (one at a time). An optical fiber (1000 $\mu \mathrm{m}$ thick, Ocean Optics, Florida, USA) was vertically placed over the samples to allow the fluorescence emission spectrum to be measured 


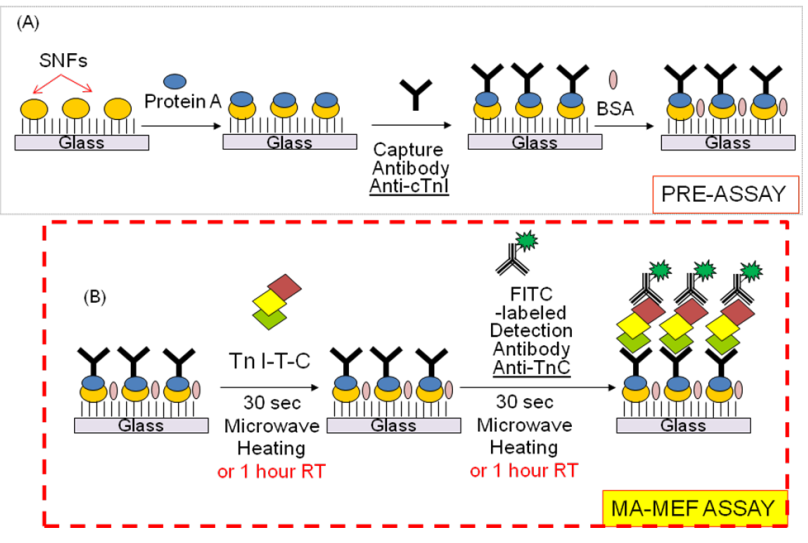

Fig. 1 Schematic depiction of the MA-MEF-based immunoassay for Tn I-T-C complex. (A) Pre-assay: where protein A, capture antibody and BSA (in buffer) attached to SNFs in sequence by incubation at room temperature for 30 minutes for each step. (B) MA-MEF assay, where Tn I-T-C (in buffer or serum) and detector antibody (in buffer) is incubated on SNFs using microwave heating for 30 seconds.

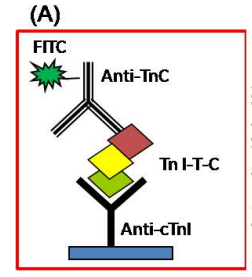

Assay in Buffer

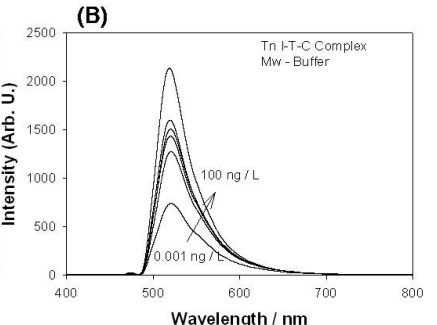

(C)

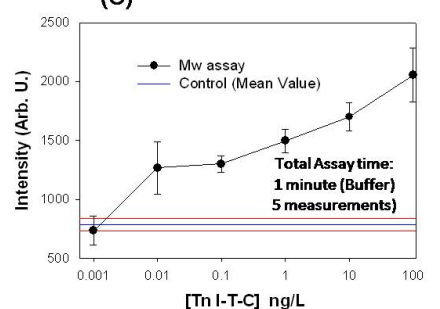

(A)

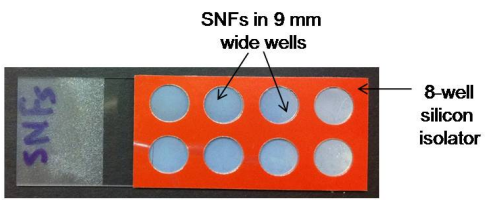

(B)

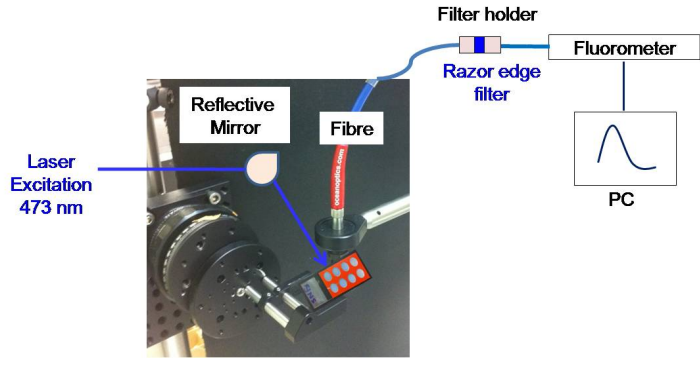

Fig. 2 (A) The real-color image of SNFs with 8-well silicon isolator that affords for the processing of 8 different samples at once. (B) The experimental geometry used for the measurement of fluorescence emission from the immunoassays.
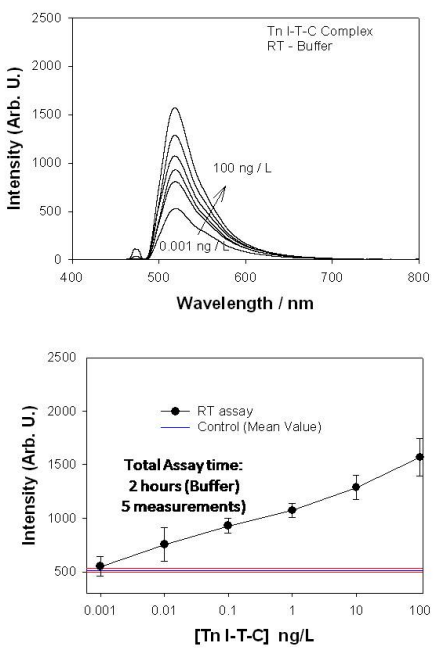

Fig. 3 (A) Schematic depiction of the Tn I-T-C immunoassay run on SNFs in buffer. (B-Left and C-Left) Tn I-T-C-concentration dependent fluorescence emission spectra. (B-Right and C-Right) Tn I-T-C concentration dependent fluorescence emission intensity at $520 \mathrm{~nm}$ from the Tn I-T-C immunoassays run in buffer at room temperature and using microwave heating.

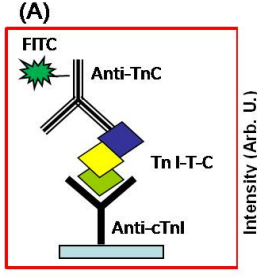

Assay in Serum
(B)

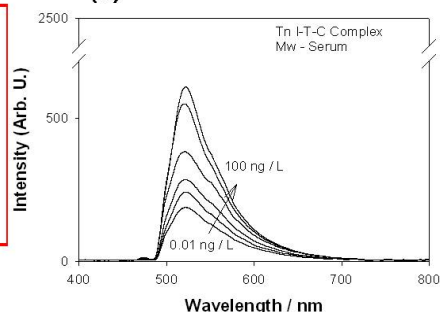

(C)

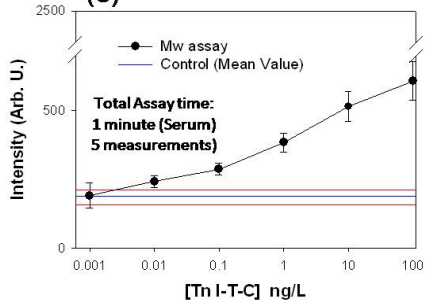

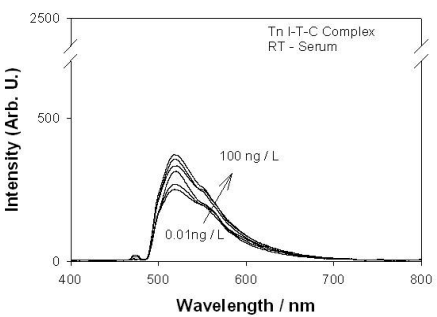

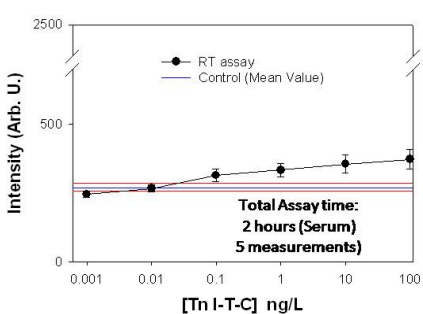

Fig. 4 (A) Schematic depiction of the Tn I-T-C immunoassay run on SNFs in serum. (B-Left and C-Left) Tn I-T-C-concentration dependent fluorescence emission spectra. (B-Right and C-Right) Tn I-T-C concentration dependent fluorescence emission intensity at $520 \mathrm{~nm}$ from the Tn I-T-C immunoassays run in serum at room temperature and using microwave heating. 
at a $45^{\circ}$ angle to the excitation source. Excitation intensity was eliminated with a $473 \mathrm{~nm}$ razor edge filter (Semrock, USA) placed in a filter holder, which couples to the optical fibers from the sample side to a spectrofluorometer (Jaz, Ocean Optics, Florida, USA). Fluorescence emission spectrum from each sample was collected using the software provided by the vendor.

\section{Results and discusion}

Fig. 3 shows the schematic depiction of the Tn I-T-C complex immunoassay run on SNFs (Fig. 3A), Tn I-T-Cconcentration dependent fluorescence emission spectra (Fig. 3B-Left and 3C-Left) and Tn I-T-C-concentration dependent fluorescence emission intensity at $520 \mathrm{~nm}$ (Fig. 3B-Right and 3C-Right) from the Tn I-T-C immunoassays run in buffer at room temperature and using microwave heating. Fluorescence emission spectra reveal that both immunoassays yield similar spectra for the range of concentrations of Tn I-T-C (1 pg ml-1 - $\left.100 \mathrm{ng} \mathrm{ml}^{-1}\right)$. The quantitative comparison of these immunoassays was made by plotting the peak emission intensity (520 $\mathrm{nm}$ ) of the spectra versus the concentration of Tn I-T-C as shown in Fig. 3B-Right and 3C-Right. These plots reveal that the both immunoassays for Tn I-T-C in buffer yields nearly similar results. The lower detection limit for the immunoassays run at room temperature and using microwave heating is determined to be $0.01 \mathrm{ng} \mathrm{ml}^{-1}$ and $0.005 \mathrm{ng} \mathrm{ml}^{-1}$, respectively. That is, one can detect lower amounts of Tn I-T-C in buffer using SNFs and microwave heating within minutes than using SNFs and room temperature incubations that takes in excess of 2 hours to complete. It is also important to note that the background emission intensity was significantly lower than the emission intensities measured for all samples. That is, the extent of non-specific binding of FITC-avidin was minimal.

After the successful demonstration that Tn I-T-C can be detected from buffer solution at room temperature and using microwave heating, the detection of Tn I-T-C in human serum at room temperature and microwave heating was carried out. We note that the results shown in Fig. 3 and Fig. 4 for Tn I-T-C immunoassay were identical with one exception: a solution of Tn I-T-C in human serum (Fig. 4) was used instead of a solution in buffer (Fig. 3). Fig. 4B-Left and 4C-Left show that the fluorescence emission spectra for the immunoassay run at room temperature and microwave heating are not the same. While there is a concentration-dependent increase in the emission spectrum for the immunoassay run using microwave heating, the emission spectra measured from immunoassay at room temperature increase slightly for all the concentrations of Tn I-T-C. This implies that the lower detection limit for Tn I-T-C in serum was much narrower compared to that of MA-MEF.

Fig. 4C-Left shows the fluorescence emission intensity peak $(520 \mathrm{~nm})$ versus Tn I-T-C concentration plot for immunoassay run using microwave heating. The emission intensities for the range of Tn I-T-C concentration were well above those measured from control experiments (a signal-to-noise ratio of $3: 1$ or above is acceptable in fluorescence measurements [8]). The emission intensities for room temperature immunoassay are close to those values of control experiments (Fig. 4C-Right). These results show that Tn I-T-C in human serum was only detectable with a lower detection level of $0.05 \mathrm{ng} \mathrm{ml}^{-1}$ and $10 \mathrm{ng} \mathrm{ml}^{-1}$ using MA-MEF technique and at room temperature, respectively.

In the following, we provide a discussion on the effect of combined use of microwave heating and SNFs in MA-MEF immunoassay scheme that affords for rapid and sensitive detection of Tn I-T-C. In MA-MEF, upon exposure of immunoassay medium placed on SNFs to microwave heating, a thermal gradient between water (or serum:buffer mixture) and SNFs is created due to the differences between their thermal conductivity $(\mathrm{k})$ values. Since $\mathrm{k}$ values for water and glass slides are similar, a thermal gradient is not expected to occur on the unsilvered part of SNFs. In the first of step of the MAMEF-based immunoassay, thermal gradient between water and SNFs results in rapid microwave-accelerated mass transfer of Tn I-T-C from the immunoassay medium to the surface of SNFs. These proteins rapidly bind to capture antibodies on the surface of SNFs. We also note that while the Fab region of the capture antibodies is oriented towards the bulk on SNFs, the Fc region is oriented towards SNFs due to binding of the Fc region with protein A. Although protein A is also present on the unsilvered part of the SNFs, since there is no thermal gradient between water and glass, it is thought that the binding of capture antibody with protein A predominantly occurs on SNFs. In the second step, where the exposure of a fresh buffer solution containing FITC-labeled detection antibody to microwave heating results in rapid microwave-accelerated mass transfer of these molecules towards the SNFs surface presenting Tn I-T-C proteins.

In addition, we note that MA-MEF technique is designed for immunoassays that employ surfaces deposited with plasmon-supporting metal nanoparticles. The sensitivity of the immunoassays carried out using glass surfaces without these metal nanoparticles were significantly less than those surfaces with metal nanoparticles.[9] This was attributed to the fact that the use of plasmon-supporting metal nanoparticles results in the increase of the fluorescence emission (metal-enhanced fluorescence) and the temperature gradient between the aqueous medium and metal surface (microwaveacceleration), as described in the Introduction section. In this regard, the use of low power microwave heating in immunoassays on glass surfaces without metal nanoparticles was not attempted in this study.

\section{4. conclusion}

The combined use of low power microwave heating and silver nanoparticle films (SNFs) deposited to glass slides in MA-MEF-based immunoassays for the detection of Tn I-T-C complex in serum samples is presented. The 
detection of Tn I-T-C complex from buffer samples was first carried out using a sandwich-type immunoassay on SNFs at room temperature (control immunoassay) and using microwave heating (MA-MEF-based immunoassay), where the lower detection limit for TnI was $0.01 \mathrm{ng} \mathrm{ml}^{-1}$ and $0.005 \mathrm{ng} \mathrm{ml}^{-1}$, respectively. The total assay time was 2 hours and 1 minute for immunoassays run at room temperature and using microwave heating, respectively. Subsequently, the detection of Tn I-T-C complex from human serum samples were carried out at room temperature and microwave heating, where Tn I-T-C complex was mixed with serum instead of buffer solutions as used in the immunoassays run for buffer samples. The use of MA-MEF technique afforded for the detection of Tn I-T-C complex from human serum samples in 1 min with a lower detection limit of $0.05 \mathrm{ng}$ $\mathrm{ml}^{-1}$, while the lower detection limit for room temperature immunoassays was $10 \mathrm{ng} \mathrm{ml}^{-1}$.

\section{Acknowledgements}

The project described was supported by Award Number 5-K25EB007565-05 from the National Institute of Biomedical Imaging and Bioengineering. The content is solely the responsibility of the authors and does not necessarily represent the official views of the National Institute of Biomedical Imaging and Bioengineering or the National Institutes of Health.

\section{References}

1. Ellenius J, Groth T, Lindahl B, Wallentin L. Early assessment of patients with suspected acute myocardial infarction by biochemical monitoring and neural network analysis. Clin Chem. 1997; 43 (10): 1919-25.

2. Panteghini M, Apple FS, Christenson RH, Dati F, Mair J, Wu AH. Use of biochemical markers in acute coronary syndromes. IFCC Scientific Division, Committee on Standardization of Markers of Cardiac Damage. International Federation of Clinical Chemistry.
Clin Chem Lab Med 1999; 37(6):687-93.http://dx.doi.org/10.1515/ CCLM.1999.107

3. (a) Gibler WB. Understanding true risk: evaluating troponins in the emergency setting. Am Heart J. 1999; 137(6):985-6,http:// dx.doi.org/10.1016/S0002-8703(99)70346-7

(b) O'Brien PJ. Blood cardiac troponin in toxic myocardial injury: archetype of a translational safety biomarker. Expert Rev Mol Diagn 2006; 6 (5):685-702,http://dx.doi.org/10.1586/14737159.6.5.685

(c) Buschmann IR, Bondke A, Elgeti T, Kuhnle Y, Dietz R, Mockel M. Positive cardiac troponin I and T and chest pain in a patient with iatrogenic hypothyroidism and no coronary artery disease. Int $J$ Cardiol 2006.

4. Heller WT, Abusamhadneh E, Finley N, Rosevear PR, Trewhella J. The solution structure of a cardiac troponin C-troponin I-troponin $\mathrm{T}$ complex shows a somewhat compact troponin $\mathrm{c}$ interacting with an extended troponin I-Troponin T component. Biochemistry. 2002; 41 (52):15654-15663.http://dx.doi.org/10.1021/bi026687c

5. Katrukha AG, Bereznikova AV, Esakova TV, Pettersson K, Lovgren T. Severina, M. E., Troponin I is released in bloodstream of patients with acute myocardial infarction not in free form but as complex. Clinical Chemistry 1997; 43 (8):1379-1385.

6. Filatov VL, Katrukha AG, Bulargina TV, Gusev NB. Troponin: Structure, properties, and mechanism of functioning. BiochemistryMoscow+ 1999; 64 (9):969-985.

7. Aslan, K.; Grell, T. A., Rapid and sensitive detection of troponin I in human whole blood samples by using silver nanoparticle films and microwave heating. Clin Chem 2011; 57 (5):746-52.http:// dx.doi.org/10.1373/clinchem.2010.159889

8. Lakowicz JR. Principles of Fluorescence Spectroscopy. 2nd ed. Kluwer Academic: New York. 1999; Vol. 2.

9. Aslan, K.; Geddes, C. D., Microwave-accelerated metal-enhanced fluorescence: Platform technology for ultrafast and ultrabright assays. Analytical Chemistry 2005; 77 (24): 8057-8067.http:// dx.doi.org/10.1021/ac0516077

Copyright:(c) $2011 \mathrm{~K}$. Aslan. This is an open-access article distributed under the terms of the Creative Commons Attribution License, which permits unrestricted use, distribution, and reproduction in any medium, provided the original author and source are credited. 